\title{
Diet quality and well-being in children and adolescents: the UP\&DOWN longitudinal study
}

\author{
Laura Esteban-Gonzalo $^{1 *}$, Anne I. Turner ${ }^{2}$, Susan J. Torres ${ }^{2}$, Irene Esteban-Cornejo ${ }^{3,4}$, \\ José Castro-Piñero ${ }^{5}$, Álvaro Delgado-Alfonso ${ }^{5}$, Ascensión Marcos ${ }^{6}$, Sonia Gómez-Martínez ${ }^{6}$ \\ and Óscar L. Veiga ${ }^{7}$ \\ ${ }^{1}$ Nursing Department, Faculty of Biomedical and Health Sciences, Universidad Europea de Madrid, 28670 Madrid, Spain \\ ${ }^{2}$ Institute for Physical Activity and Nutrition, School of Exercise and Nutrition Sciences, Deakin University, Geelong, \\ VIC 3220, Australia \\ ${ }^{3}$ Department of Psychology, Center for Cognitive and Brain Health, Northeastern University, Boston, MA O2115, USA \\ ${ }^{4}$ PROFITH "PROmoting FITness and Health through physical activity" research group, Department of Physical Education and \\ Sports, Faculty of Sport Sciences, University of Granada, 18071 Granada, Spain \\ ${ }^{5}$ Department of Physical Education, Faculty of Education Sciences, University of Cadiz, 11519 Puerto Real, Spain \\ ${ }^{6}$ Institute of Food Science, Technology and Nutrition (ICTAN), Spanish National Research Council (CSIC), \\ 28040 Madrid, Spain \\ ${ }^{7}$ Department of Physical Education, Sports and Human Movement, Autonomous University of Madrid, 28049 Madrid, Spain
}

(Submitted 12 April 2018 - Final revision received 24 September 2018 - Accepted 26 September 2018 - First published online 5 November 2018)

\section{Abstract}

The present study examined the association between high-quality diet (using the Mediterranean diet (MD) as an example) and well-being cross-sectionally and prospectively in Spanish children and adolescents. Participants included 533 children and 987 adolescents at baseline and 527 children and 798 adolescents at 2-year follow-up, included in the UP\&DOWN study (follow-up in schoolchildren and adolescents with and without Down's syndrome). The present study excluded participants with Down's syndrome. Adherence to an MD was assessed using the KIDMED index. Well-being was measured using the Positive and Negative Affect Schedule and the KIDSCREEN-10 questionnaire. Associations between MD adherence and well-being were assessed using multi-level, mixed-effects linear regression. At baseline, MD adherence was positively related to health-related quality of life in secondary school girls and boys $(\beta=0 \cdot 41$, sE $0 \cdot 10, P<0 \cdot 001$; $\beta=0 \cdot 46$, sE $0 \cdot 10, P<0 \cdot 001$, respectively) and to positive affect in secondary school girls and boys $(\beta=0 \cdot 16, \mathrm{sE} 0 \cdot 05, P=0 \cdot 006 ; \beta=0 \cdot 20$, sE $0 \cdot 05, P<0 \cdot 001$, respectively) and in primary school boys $(\beta=0 \cdot 20$, se $0 \cdot 08, P=0 \cdot 019)$. At 2 -year follow-up, MD adherence was negatively related to negative affect in secondary school adolescent girls and boys $(\beta=-0 \cdot 15$, se $0 \cdot 07, P=0 \cdot 047 ; \beta=-0 \cdot 16$, SE $0 \cdot 06, P=0 \cdot 019$, respectively), and MD adherence was associated with higher positive affect scores in secondary school girls $(\beta=0 \cdot 30$, sE $0 \cdot 06, P<0 \cdot 001)$ and in primary school boys $(\beta=0 \cdot 20$, se $0 \cdot 09, P=0 \cdot 023)$. However, MD adherence at baseline did not predict well-being indicators at 2 -year follow-up. In conclusion, higher MD adherence was found to behave as a protective factor for positive well-being in cross-sectional analysis.

Key words: Diet quality: Mediterranean diet: Well-being: Quality of life: Children: Adolescents

In recent years, interest in well-being has increased in the Western societies due to its relationship with physical health and survival ${ }^{(1)}$; however, previous studies have addressed signs of a deteriorated well-being in youths. Mental health disorders are one of the most common chronic health conditions in young people and represent a significant economic burden. One fourth of adolescents report a mental health disorder in the previous year and a third across their lifetimes ${ }^{(2)}$. Childhood and adolescence are periods of critical importance for understanding the development of well-being and the contribution to behavioural risk.

Well-being is a complex aspect of life, whereby understanding and measuring well-being are challenges for scientists. On one hand, a subjective psychological dimension of wellbeing has to be considered. Subjective well-being is defined as someone's evaluation of his or her own life, including emotional reactions and feelings of satisfaction ${ }^{(3)}$. An important component of subjective well-being is described as hedonic

Abbreviations: HR-QoL, health-related quality of life; MD, Mediterranean diet; SES, socio-economic status.

* Corresponding author: L. Esteban-Gonzalo, email laura.esteban@universidadeuropea.es 
well-being, an affective indicator that distinguishes between positive and negative affect as independent measures, considering feelings such as happiness, sadness, anger, stress and pain $^{(4)}$. On the other hand, well-being related to quality of life should be regarded. A commonly used measure is healthrelated quality of life (HR-QoL), which refers to an individual's perception and subjective evaluation of their health and wellbeing within their unique cultural environment ${ }^{(5)}$.

Diet quality is a behavioural protective factor that has been associated with well-being and mental health in adolescents, according to the literature. In this sense, a recent systematic review of observational studies found an association between a healthy diet and lower levels of depression ${ }^{(6)}$. An example of a high-quality dietary pattern is the Mediterranean diet (MD), which is characterised by the consumption of fish or seafood, white meat, moderate amounts of dairy products, increased amounts of enriched fibre foods (such as fruits, vegetables, legumes, nuts and whole grain cereals) and unsaturated fats, especially olive oil ${ }^{(7)}$. Its protective effect on mental ${ }^{(8-10)}$ and cardiovascular and metabolic disease ${ }^{(11,12)}$ has been reported previously. Adherence to an MD or a high-quality diet has been associated with better HR-QoL in Mediterranean ${ }^{(13,14)}$ and nonMediterranean adolescents ${ }^{(15,16)}$. Furthermore, the higher adherence to MD was associated with a higher life satisfaction status in Spanish adolescents ${ }^{(17)}$.

Therefore, since none of the previously described studies had evaluated the longitudinal relationship of MD adherence and well-being in children and adolescents, the aims of the present study were (1) to analyse the cross-sectional relationship between MD adherence and well-being indicators in a large sample of Spanish children and adolescents at baseline and at 2-year follow-up and (2) to assess the potential predictive effect of MD adherence at baseline on well-being indicators measured at 2-year follow-up.

\section{Methods}

\section{Study design}

This study was performed under the umbrella of the UP\&DOWN study (follow-up in schoolchildren and adolescents with and without Down's syndrome: psycho-environmental and genetic determinants of physical activity and their impact on physical fitness, CVD, inflammatory biomarkers and mental health). Data were collected on MD adherence (KIDMED index, a Mediterranean diet quality index for children and teenagers) and wellbeing indicators (Positive and Negative Affect Schedule (PANAS) and HR-QOL). The present study excluded children and adolescents with Down's syndrome and instead included only those without Down's syndrome. Detailed information about the study design has been reported previously ${ }^{(18)}$.

\section{Participants}

Participants were recruited from primary schools in Cadiz and secondary schools in Madrid (Spain). Data were compiled over a daily school class from September to June: (a) baseline data from 2011 to 2012 and (b) 2-year follow-up from 2013 to 2014.
Participants' selection criteria for children and adolescents considered in the study were as follows: (i) to study in first/ fourth grades (6-7 and 9-10 years old, respectively) for children and seventh/tenth grades (12-13 and 15-16 years old, respectively) for adolescents at baseline and (ii) to not have physical disability or health problems, which might limit the levels of physical activity. For the present study, primary schoolchildren (aged between 6 and 11 years old at baseline) and secondary school adolescents (aged between 11 and 19 years old at baseline) with valid data regarding MD adherence and well-being indicators were included. Thus, a total of 536 primary schoolchildren (50\% girls) and 987 secondary school adolescents ( $49 \%$ girls) provided valid data at baseline, while 527 children (50\% girls) and 798 adolescents (49\% girls) provided valid data at 2-year follow-up. Cross-sectional analyses included all participants who participated as each time point, whereas longitudinal analyses included only those participants that provided valid data at both baseline and 2-year follow-up.

Before participating in the UP\&DOWN study, parents and school supervisors were informed by letter about the purpose of the study. Written parental consent and child/adolescent assent were obtained. The UP\&DOWN study was approved by the Ethics Committee of the Hospital Puerta de Hierro in Madrid, the Bioethics Committee of the Spanish National Research Council and the Ethics Committee for Research Involving Human Subjects at Cádiz University.

\section{Mediterranean diet adherence}

Mediterranean diet adherence was assessed using the KIDMED index ${ }^{(19)}$. This is an MD quality index, ranging from -4 to 12 and based on a 16 yes-or-no question test, which determines adherence to the Mediterranean dietary pattern. Questions with a negative connotation were scored -1 , while questions with positive connotation were scored 1 . Higher scores indicate higher adherence to the $\mathrm{MD}^{(19)}$. The KIDMED index has shown good internal consistency with a Cronbach's $\alpha$ coefficient of $0 \cdot 7^{(20)}$.

\section{Well-being}

Hedonic subjective psychological well-being was measured using the PANAS ${ }^{(4,21)}$. This instrument comprises two 10-item scales, providing independent measurements of positive and negative affect, which are linked to positive feelings such as joy or pleasure and negative feelings such as anxiety and sadness, respectively. Participants are required to respond to every item using a five-point Likert-type scale that ranges from very slightly or not at all to extremely. The scoring range is from 10 to 30 , where higher scores indicate greater positive or negative feelings ${ }^{(4)}$. In the current study, the Spanish version for children and adolescents (PANASN) was employed, respecting the same bi-dimensional structure and showing adequate internal consistency as well as convergent and discriminant validity (for the positive affect scale, the Cronbach's $\alpha$ coefficient was 0.87-0.89 and for the negative affect scale it was 0.89-0.91) ${ }^{(21)}$.

HR-QoL was assessed using the KIDSCREEN-10 (a healthrelated quality of life questionnaire for children and young people and their parents), which provides a self-reported 
well-being and HR-QoL measure for children and adolescents $^{(22)}$. It is composed of ten items answered through a fivepoint response scale (items 1 and 9: not at all-slightly-moderately-very-extremely; all others: never-seldom-quite oftenvery often-always). As a result, the score ranges from 10 to 50, where higher scores indicate better HR-QoL. The ten items explore a wide range of factors related to well-being and quality of life, such as physical activity, energy and fitness, depressive and stressful feelings, opportunities to structure and enjoy social life and leisure time, participation in social activities, quality of social interactions, perception of his or her cognitive capacity and satisfaction with school performance. This tool has demonstrated adequate reliability, validity and internal consistency with a Cronbach's $\alpha$ coefficient of $0 \cdot 87^{(23)}$.

\section{Covariates}

Covariates were included to adjust for confounding by variables likely to be associated with both diet and well-being. The covariates included were age, socio-economic status (SES), overweight and obesity prevalence as well as pubertal staging.

SES was measured using the family affluence scale, a fouritem scale ${ }^{(24)}$. This score was obtained after summing the answers provided by the participants in the four questions. Based on the score obtained, children and adolescents were categorised into low (from 0 to 2), medium (from 3 to 5) or high SES (from 6 to 8 ) ${ }^{(25)}$

Weight and height were measured using standard procedures $^{(18)}$. Weight was measured using an electronic scale (SECA 701; range, 0.05-220 kg; precision, $0.05 \mathrm{~kg}$ ) and height was measured in the Frankfort plane using a telescopic heightmeasuring instrument (SECA 220; range, $85-200 \mathrm{~cm}$; precision, $1 \mathrm{~mm})$. BMI was calculated as weight/height squared $\left(\mathrm{kg} / \mathrm{m}^{2}\right)$. BMI, age- and sex-specific cut offs proposed by Cole et al. ${ }^{(26)}$ were used to categorise primary and secondary school girls and boys as normal weight, underweight and overweight or obese.

Self-reported pubertal staging of primary and secondary school girls and boys was measured according to Tanner scale $^{(27)}$. This scale includes two separate measures for girls and boys. For girls, pubic hair distribution and breast development were self-reported. For boys, pubic hair distribution and genital development were self-reported. Although self-reported pubertal assessment is not a reliable measure for exact pubertal staging, it has been frequently used in epidemiological studies due to its accuracy for a simple distinction between pre-puberty and puberty ${ }^{(27)}$. However, since pubertal staging showed no effect on the associations examined, it was not included in the adjusted model.

\section{Statistical analysis}

All statistical analyses were conducted using the Statistical Package for the Social Sciences software version 21.0 (SPSS Inc.) and STATA/SE 14.1 software (StataCorp LP).

Descriptive statistics (mean values and standard deviations or numbers and percentages) were calculated to describe the participant characteristics. Differences between primary and secondary school girls and boys were analysed by Student's $t$ test for continuous variables and $\chi^{2}$ test for categorical variables. Differences between values at baseline and at 2 -year follow-up were analysed by paired $t$ test.

Multi-level, mixed-effects linear regression was used to test the association between MD adherence and well-being indicators. Interactions for sex and age were significant, so analyses were conducted separately for primary school (aged 6-11 years) and secondary school (aged 11-19 years) girls and boys. All models included a random intercept for school and class. In cross-sectional analysis, unadjusted models and models adjusting for age, SES and overweight and obesity prevalence were fitted. In longitudinal analysis, models adjusted for baseline well-being indicators (model 1) and then additionally adjusted for 2-year follow-up age, SES, overweight and obesity prevalence (model 2) were fitted. Covariates were included one-by-one in the models for each analysis performed (data not shown, given the lack of effect of the mentioned variables).

\section{Results}

Characteristics of the primary and secondary school girls and boys are presented in Table 1. At baseline, compared to secondary school girls, primary school girls showed lower SES, pubertal stage, higher prevalence of overweight and obesity and higher KIDSCREEN and positive affect scores (all $P<0.001)$. Furthermore, compared to secondary school boys, primary school boys showed lower SES, lower pubertal stage, lower KIDMED and KIDSCREEN scores and higher positive and negative affect scores (all $P<0 \cdot 001$ ). At 2-year follow-up, primary school girls had a lower pubertal stage, greater KIDSCREEN scores and lower negative affect scores compared to secondary school girls (all $P<0.001$ ), and primary school boys had lower pubertal stage $(P<0 \cdot 001)$ and KIDMED scores $(P=0 \cdot 019)$, and higher KIDSCREEN scores $(P=0.001)$ than secondary school boys.

Multi-level, mixed-effects linear regression models for the cross-sectional association between MD adherence and wellbeing at baseline are presented in Table 2. MD adherence was positively associated with KIDSCREEN scores in secondary school girls and boys, and this persisted after adjusting for relevant covariates (age, SES and prevalence of overweight and obesity). A unit increase in MD adherence score was associated with an increase in KIDSCREEN scores in secondary school adolescent girls and boys $(\beta=0.41$, sE $0 \cdot 10, P<0 \cdot 001 ; \beta=0.46$, SE $0 \cdot 10, P<0 \cdot 001$, respectively). A one-unit increase in MD adherence score was also associated with an increase in positive affect scores in secondary school girls and boys $(\beta=0 \cdot 16$, SE $0 \cdot 05$, $P=0 \cdot 006 ; \beta=0 \cdot 20$, sE $0 \cdot 05, P<0 \cdot 001$, respectively) and in primary school boys $(\beta=0 \cdot 20$, se $0 \cdot 08, P=0 \cdot 019)$. There was no relationship between MD adherence and negative affect scores.

Changes in characteristics over 2-year follow-up are also presented in Table 1. Pubertal stage increased in all primary and secondary girls and boys after 2 years $(P<0.001$ for all). In primary school girls, KIDMED, KIDSCREEN, positive and negative affect scores all reduced after 2-year follow-up (all $P<0.001)$. In secondary school girls, KIDSCREEN and positive affect scores reduced (both $P<0 \cdot 001$ ), whereas 
Table 1. Characteristics of primary and secondary school girls and boys at baseline and 2-year follow-up

(Mean values and standard deviations)

\begin{tabular}{|c|c|c|c|c|c|c|c|c|c|c|}
\hline & \multicolumn{4}{|c|}{ Girls } & \multirow[b]{3}{*}{$P+$} & \multicolumn{4}{|c|}{ Boys } & \multirow[b]{3}{*}{$P \dagger$} \\
\hline & \multicolumn{2}{|c|}{ Primary school } & \multicolumn{2}{|c|}{ Secondary school } & & \multicolumn{2}{|c|}{ Primary school } & \multicolumn{2}{|c|}{ Secondary school } & \\
\hline & Mean & SD & Mean & SD & & Mean & SD & Mean & SD & \\
\hline \multicolumn{11}{|l|}{ Characteristics at baseline } \\
\hline$n$ & & & & & & & & & & \\
\hline Age (years) & $8 \cdot 2$ & 1.5 & $14 \cdot 1$ & 1.6 & $<0.001$ & 8.0 & 1.5 & $14 \cdot 0$ & 1.6 & $<0.001$ \\
\hline Prevalence overweight/obesity (\%) & & & & & $<0.001$ & & & & & 0.243 \\
\hline SES & & & & & $<0.001$ & & & & & $<0.001$ \\
\hline Low (\%) & & & & & & & & & & \\
\hline Medium (\%) & & & & & & & & & & \\
\hline High (\%) & & & & & & & & & & \\
\hline Tanner score $1 \ddagger(1-5)$ & 1.3 & 0.5 & 3.3 & 0.8 & $<0.001$ & 1.4 & 0.5 & 3.4 & 0.9 & $<0.001$ \\
\hline Tanner score $2 \S(1-5)$ & 1.5 & 0.6 & 3.4 & 0.7 & $<0.001$ & 1.5 & 0.6 & 3.7 & 0.9 & $<0.001$ \\
\hline KIDMED scores ( -4 to 12$)$ & 6.5 & $2 \cdot 2$ & $6 \cdot 4$ & $2 \cdot 2$ & 0.497 & 5.9 & 2.4 & 6.6 & 2.3 & $<0.001$ \\
\hline KIDSCREEN scores (10-50) & $42 \cdot 0$ & $5 \cdot 0$ & $38 \cdot 1$ & 5.3 & $<0.001$ & 41.5 & 4.5 & 39.6 & $5 \cdot 2$ & $<0.001$ \\
\hline Positive affect scores $(10-30)$ & $25 \cdot 2$ & 3.1 & $24 \cdot 1$ & 3.0 & $<0.001$ & 24.7 & 2.9 & 23.8 & 2.9 & $<0.001$ \\
\hline Negative affect scores $(10-30)$ & $17 \cdot 7$ & $3 \cdot 3$ & $17 \cdot 7$ & $3 \cdot 2$ & 0.738 & $18 \cdot 1$ & 3.5 & $16 \cdot 4$ & 3.2 & $<0.001$ \\
\hline \multicolumn{11}{|l|}{ Characteristics at 2-year follow-up } \\
\hline$n$ & & & & & & & & & & \\
\hline Tanner score $1(1-5)$ & $1 \cdot 8^{\star \star *}$ & 0.9 & $3 \cdot 7^{\star \star \star}$ & 0.6 & $<0.001$ & $1.9^{* \star \star}$ & 0.7 & $4 \cdot 0^{\star \star \star}$ & 0.7 & $<0.001$ \\
\hline Tanner score $2(1-5)$ & $2 \cdot 1^{\star \star \star}$ & 0.9 & $3 \cdot 9^{\star \star \star}$ & 0.6 & $<0.001$ & $2 \cdot 3^{\star \star \star}$ & 0.7 & $4 \cdot 4^{\star \star \star}$ & 0.6 & $<0.001$ \\
\hline KIDMED scores ( -4 to 12$)$ & $6 \cdot 0^{\star \star *}$ & 2.4 & $6 \cdot 3$ & $2 \cdot 3$ & 0.726 & $6 \cdot 1$ & 2.4 & $6 \cdot 6^{*}$ & 2.4 & 0.019 \\
\hline KIDSCREEN scores $(10-50)$ & $38 \cdot 8^{\star \star \star}$ & 6.4 & $35 \cdot 5^{\star \star \star}$ & 5.5 & $<0.001$ & $39 \cdot 2^{\star \star \star}$ & 5.7 & $37 \cdot 7^{* \star *}$ & $5 \cdot 2$ & 0.001 \\
\hline Positive affect scores $(10-30)$ & $23 \cdot 5^{\star \star \star}$ & 3.4 & $23 \cdot 4^{\star \star \star}$ & 3.1 & 0.739 & $23 \cdot 1^{\star \star \star}$ & 3.7 & $22 \cdot 9^{\star \star \star}$ & 3.1 & 0.356 \\
\hline Negative affect scores $(10-30)$ & $16 \cdot 3^{\star \star \star}$ & 3.3 & $18 \cdot 1^{*}$ & 3.4 & $<0.001$ & $15 \cdot 9^{\star \star \star}$ & 3.5 & $16 \cdot 3$ & 3.4 & 0.139 \\
\hline \multicolumn{11}{|l|}{ Changes over 2-year follow-up } \\
\hline KIDMED scores ( -4 to 12$)$ & -0.6 & 2.5 & -0.2 & $2 \cdot 1$ & 0.035 & -0.2 & 2.8 & -0.2 & $2 \cdot 4$ & 0.798 \\
\hline KIDSCREEN scores & $-3 \cdot 3$ & 6.7 & -2.5 & 5.9 & 0.155 & $-2 \cdot 3$ & $6 \cdot 4$ & -1.9 & 5.5 & 0.419 \\
\hline Positive affect scores (10-30) & -1.6 & $4 \cdot 2$ & -0.7 & 3.4 & 0.008 & -1.4 & 4.0 & -0.9 & 3.3 & 0.128 \\
\hline Negative affect scores $(10-30)$ & -1.3 & 4.2 & 0.4 & 3.4 & $<0.001$ & $-2 \cdot 2$ & 4.4 & -0.1 & 3.5 & $<0.001$ \\
\hline
\end{tabular}

SES, socio-economic status.

Wifferent from that at baseline: * $P<0.05, * * * P<0.001$ (paired $t$ tests)

† Student's $t$ test (Pearson $x^{2}$ test for prevalence of overweight/obesity and SES).

$\S$ Genitals/breasts development. 
Table 2. Multi-level, mixed-effect linear regression models for the cross-sectional association between Mediterranean diet adherence (KIDMED scores) and well-being (KIDSCREEN, positive and negative affect scores) at baseline in Spanish primary and secondary school girls and boys

( $\beta$-Coefficients with their standard errors; numbers and $95 \%$ confidence intervals)

\begin{tabular}{|c|c|c|c|c|c|c|c|c|c|c|}
\hline & \multicolumn{4}{|c|}{ Unadjusted model } & \multirow[b]{2}{*}{$P$} & \multicolumn{4}{|c|}{ Adjusted model ${ }^{*}$} & \multirow[b]{2}{*}{$P$} \\
\hline & $n$ & $\beta$ & SE & $95 \% \mathrm{Cl}$ & & $n$ & $\beta$ & SE & $95 \% \mathrm{Cl}$ & \\
\hline \multicolumn{11}{|l|}{ KIDSCREEN scores } \\
\hline \multicolumn{11}{|l|}{ Girls } \\
\hline Primary school & 246 & 0.07 & 0.14 & $-0.21,0.35$ & 0.628 & 241 & 0.08 & 0.14 & $-0.19,0.37$ & 0.547 \\
\hline Secondary school & 467 & 0.49 & 0.10 & $0.29,0.69$ & $<0.001$ & 462 & 0.41 & 0.10 & $0.21,0.61$ & $<0.001$ \\
\hline \multicolumn{11}{|l|}{ Boys } \\
\hline Primary school & 249 & 0.14 & 0.12 & $-0.09,0.38$ & 0.231 & 238 & 0.11 & 0.12 & $-0.13,0.36$ & 0.361 \\
\hline Secondary school & 492 & 0.45 & 0.10 & $0.24,0.65$ & $<0.001$ & 486 & 0.46 & 0.10 & $0.25,0.66$ & $<0.001$ \\
\hline \multicolumn{11}{|l|}{ Positive affect scores } \\
\hline \multicolumn{11}{|l|}{ Girls } \\
\hline Primary school & 251 & 0.10 & 0.09 & $-0.07,0.28$ & 0.263 & 244 & 0.10 & 0.09 & $-0.07,0.28$ & 0.249 \\
\hline Secondary school & 470 & 0.17 & 0.05 & $0.05,0.29$ & 0.003 & 465 & 0.16 & 0.05 & $0.04,0.28$ & 0.006 \\
\hline \multicolumn{11}{|l|}{ Boys } \\
\hline Primary school & 251 & 0.23 & 0.08 & $0.07,0.40$ & 0.004 & 237 & 0.20 & 0.08 & $0.03,0.37$ & 0.019 \\
\hline Secondary school & 495 & 0.19 & 0.05 & $0.08,0.30$ & 0.001 & 489 & 0.20 & 0.05 & $0.09,0.32$ & $<0.001$ \\
\hline \multicolumn{11}{|l|}{ Negative affect scores } \\
\hline \multicolumn{11}{|l|}{ Girls } \\
\hline Primary school & 251 & -0.09 & 0.09 & $-0.28,0.10$ & 0.358 & 244 & -0.09 & 0.09 & $-0.28,0.09$ & 0.338 \\
\hline Secondary school & 469 & -0.10 & 0.06 & $-0.23,0.01$ & 0.098 & 464 & -0.08 & 0.06 & $-0.21,0.04$ & 0.199 \\
\hline \multicolumn{11}{|l|}{ Boys } \\
\hline Primary school & 253 & -0.03 & 0.09 & $-0.22,0.14$ & 0.678 & 239 & -0.02 & 0.10 & $-0.22,0.17$ & 0.806 \\
\hline Secondary school & 495 & -0.11 & 0.06 & $-0.23,0.01$ & 0.082 & 489 & -0.11 & 0.06 & $-0.24,0.00$ & 0.068 \\
\hline
\end{tabular}

$\beta$, Unstandardised coefficient.

* Adjusted model: analyses were adjusted for age, socio-economic status and prevalence of overweight and obesity (all collected at baseline). 
negative affect scores increased $(P<0.05)$ after 2 -year followup. In primary school boys, KIDSCREEN, positive and negative affect scores all reduced after 2 -year follow-up (all $P<0.001$ ). In secondary school boys, KIDMED $(P<0.05)$, KIDSCREEN, positive and negative affect scores all reduced (all $P<0 \cdot 001$ ) after 2-year of follow-up.

Multi-level, mixed-effect linear regression models for the cross-sectional association between MD adherence and wellbeing at 2-year follow-up are presented in Table 3. A one-unit increase in MD adherence score was associated with increased KIDSCREEN scores in secondary school girls in an unadjusted model $(\beta=0 \cdot 27$, se $0 \cdot 12, P=0 \cdot 025)$. This association was no longer significant after adjustment for age, SES and overweight/ obesity. On the other hand, a one-unit increase in MD adherence score was associated with increased positive affect scores in secondary school girls in the adjusted model $(\beta=0.30$, SE $0.06, P<0.001)$ and in primary school boys $(\beta=0.20$, sE 0.09 , $P=0.023)$. Finally, a one-unit increase in MD adherence score was related to a decrease in negative affect scores in secondary school adolescent girls and boys in the adjusted model $(\beta=-0.15$, SE $0.07, \quad P=0.047 ; \quad \beta=-0.16$, SE $0.06, \quad P=0.019$, respectively).

Multi-level, mixed-effect linear regression models for the longitudinal association between MD adherence at baseline and well-being indicators (at 2-year follow-up) are shown in Table 4. After adjusting for baseline levels of well-being indicators, MD adherence at baseline did not predict well-being indicators at 2-year follow-up, either before (model 1) or after controlling for age, SES, prevalence of overweight and obesity (model 2).

In addition, not only age and SES but also pubertal staging was included one-by-one in every model performed and did not show any effect in any of the associations examined (data not shown).

\section{Discussion}

This study found that a higher MD adherence was associated with better HR-QoL and positive affect in secondary school girls and boys at baseline. In addition, MD adherence was associated with greater levels of positive affect in primary school boys and secondary school girls and lower levels of negative affect in secondary school adolescent girls and boys at 2-year follow-up. However, we found no prospective associations between MD adherence at baseline and well-being indicators at 2-year follow-up after controlling for baseline levels of well-being indicators.

Our cross-sectional findings about the association between higher MD adherence and better HR-QOL is consistent with a number of other studies. For example, there was a positive association between adherence to the MD and HR-QOL in Spanish adolescents living in Granada ${ }^{(14)}$ and Spanish adolescents living in rural and urban areas who reported higher adherence to the MD and a greater life satisfaction ${ }^{(17)}$. Also, a study conducted in Greece reported a positive association between adherence to MD and HR-QoL in male and female adolescents $^{(13)}$. Furthermore, in non-Mediterranean countries, a similar association has been found, where better diet quality was related to higher HR-QoL in urban and rural Australian adolescents $^{(15)}$ as well as in socially disadvantaged New Zealand adolescents ${ }^{(16)}$.

In the present study, we did not see longitudinal associations between baseline MD adherence and HR-QoL after 2-year follow-up, although borderline negative associations were observed between MD adherence and negative affect in secondary school boys and girls. In this regard, we have not found any other longitudinal studies analysing this association in Spanish or Mediterranean adolescent populations. To our knowledge, the only longitudinal study carried out in Spain reported a positive association between adherence to the MD and HR-QoL in an adult population ${ }^{(28)}$. In addition, studies conducted in non-Mediterranean populations found evidence of a longitudinal association between high-quality diets and a more favourable HR-QoL in older adults ${ }^{(29)}$. For example, a study carried out in an older Australian population found greater adherence to a healthy diet to be associated with a more favourable HR-QoL 2 years later in men and women aged 55-65 years $^{(30)}$

\section{Differences in the cross-sectional association of Mediterranean diet adherence and well-being indicators between baseline and 2-year follow-up}

In the present study, we found that higher MD adherence was associated with better HR-QOL and positive affect in secondary school girls and boys at baseline and lower negative affect scores in secondary school adolescent girls and boys at 2-year follow-up. These variations observed in the associations examined between baseline and 2-year follow-up may be related to changes experienced by the participants between childhood and adolescence. From baseline to 2-year follow-up, the change in age of boys and girls in both the groups (Table 1) was reflected in significant increases in self-reported Tanner staging (Table 1). However, the influence of these changes in biological maturation was tested and discarded due to the variable showing no influence on the associations examined. We could only speculate that critical times during childhood and adolescence could bring potential differences in psychological maturity (that were not measured in this study), which may influence the relationships between MD adherence and well-being indicators. Furthermore, sex and age differences in well-being indictors have been widely described in the literature ${ }^{(31,32)}$ and could also be involved in the variations observed in the relationship of MD adherence and well-being between baseline and 2-year follow-up.

\section{Changes in well-being indicators and Mediterranean diet adherence along 2-year follow-up}

In the present study, we found that HR-QoL and positive affect scores decreased and negative affect scores increased in primary and secondary school girls and boys, except negative affect scores improved in secondary school girls only, indicating that overall well-being had deteriorated over the 2 years of the 
Table 3. Multi-level, mixed-effect linear regression models for the cross-sectional association between Mediterranean diet adherence (KIDMED scores) and well-being (KIDSCREEN, positive and negative affect scores) at 2-year follow-up in Spanish primary and secondary school girls and boys ( $\beta$-Coefficients with their standard errors; numbers and $95 \%$ confidence intervals)

\begin{tabular}{|c|c|c|c|c|c|c|c|c|c|c|}
\hline & \multicolumn{4}{|c|}{ Unadjusted model } & \multirow[b]{2}{*}{$P$} & \multicolumn{4}{|c|}{ Adjusted model ${ }^{*}$} & \multirow[b]{2}{*}{$P$} \\
\hline & $n$ & $\beta$ & SE & $95 \% \mathrm{Cl}$ & & $n$ & $\beta$ & SE & $95 \% \mathrm{Cl}$ & \\
\hline \multicolumn{11}{|l|}{ KIDSCREEN scores } \\
\hline \multicolumn{11}{|l|}{ Girls } \\
\hline Primary school & 238 & 0.20 & 0.17 & $-0.13,0.55$ & 0.239 & 229 & 0.11 & 0.18 & $-0.23,0.47$ & 0.519 \\
\hline Secondary school & 374 & 0.27 & 0.12 & $0.03,0.51$ & 0.025 & 372 & 0.17 & 0.12 & $-0.06,0.41$ & 0.147 \\
\hline \multicolumn{11}{|l|}{ Boys } \\
\hline Primary school & 256 & 0.24 & 0.14 & $-0.04,0.52$ & 0.096 & 243 & 0.24 & 0.14 & $-0.04,0.53$ & 0.092 \\
\hline Secondary school & 389 & 0.13 & 0.10 & $-0.06,0.34$ & 0.183 & 389 & 0.09 & $0 \cdot 10$ & $-0.11,0.29$ & 0.376 \\
\hline \multicolumn{11}{|l|}{ Positive affect scores } \\
\hline \multicolumn{11}{|l|}{ Girls } \\
\hline Primary school & 240 & 0.09 & 0.10 & $-0.11,0.30$ & 0.376 & 231 & 0.02 & 0.09 & $-0.16,0.21$ & 0.808 \\
\hline Secondary school & 376 & 0.33 & 0.06 & $0.19,0.46$ & $<0.001$ & 374 & 0.30 & 0.06 & $0.16,0.43$ & $<0.001$ \\
\hline \multicolumn{11}{|l|}{ Boys } \\
\hline Primary school & 255 & 0.20 & 0.09 & $0.02,0.38$ & 0.027 & 241 & 0.20 & 0.09 & $0.02,0.38$ & 0.023 \\
\hline Secondary school & 390 & 0.06 & 0.06 & $-0.06,0.18$ & 0.338 & 390 & 0.04 & 0.06 & $-0.07,0.16$ & 0.482 \\
\hline \multicolumn{11}{|l|}{ Negative affect scores } \\
\hline \multicolumn{11}{|l|}{ Girls } \\
\hline Primary school & 244 & -0.16 & 0.09 & $-0.34,0.01$ & 0.073 & 234 & -0.14 & 0.09 & $-0.33,0.04$ & 0.138 \\
\hline Secondary school & 376 & -0.16 & 0.07 & $-0.31,-0.02$ & 0.025 & 374 & -0.15 & 0.07 & $-0.30,-0.02$ & 0.047 \\
\hline \multicolumn{11}{|l|}{ Boys } \\
\hline Primary school & 255 & -0.03 & 0.09 & $-0.21,0.14$ & 0.707 & 248 & -0.02 & 0.09 & $-0.19,0.15$ & 0.810 \\
\hline Secondary school & 390 & -0.16 & 0.06 & $-0.31,-0.29$ & 0.015 & 390 & -0.16 & 0.06 & $-0.29,-0.02$ & 0.019 \\
\hline
\end{tabular}

$\beta$, Unstandardised coefficient.

* Adjusted model: analyses were adjusted for age, socio-economic status and prevalence of overweight and obesity (all collected at 2-year follow-up). 
Table 4. Multi-level, mixed-effect linear regression models for the longitudinal association between Mediterranean diet adherence at baseline (KIDMED scores) and well-being at 2-year follow-up (KIDSCREEN, positive and negative affect scores) in Spanish primary and secondary school girls and boys

( $\beta$-Coefficients with their standard errors; numbers and $95 \%$ confidence intervals)

\begin{tabular}{|c|c|c|c|c|c|c|c|c|c|c|}
\hline & \multicolumn{4}{|c|}{ Model $1^{*}$} & \multirow[b]{2}{*}{$P$} & \multicolumn{4}{|c|}{ Model $2 \dagger$} & \multirow[b]{2}{*}{$P$} \\
\hline & $n$ & $\beta$ & SE & $95 \% \mathrm{Cl}$ & & $n$ & $\beta$ & $\mathrm{SE}$ & $95 \% \mathrm{Cl}$ & \\
\hline \multirow{2}{*}{\multicolumn{11}{|c|}{ KIDSCREEN scores }} \\
\hline \multicolumn{9}{|l|}{ Girls } & & \\
\hline Primary school & 218 & 0.20 & 0.19 & $-0.17,0.57$ & 0.295 & 211 & 0.18 & 0.18 & $-0.18,0.54$ & 0.328 \\
\hline Secondary school & 366 & 0.04 & 0.11 & $-0.18,0.25$ & 0.707 & 364 & 0.01 & 0.11 & $-0.21,0.24$ & 0.884 \\
\hline \multicolumn{11}{|l|}{ Boys } \\
\hline Primary school & 237 & 0.05 & 0.16 & $-0.26,0.37$ & 0.738 & 227 & 0.07 & 0.16 & $-0.25,0.40$ & 0.655 \\
\hline Secondary school & 386 & 0.00 & 0.10 & $-0.20,0.21$ & 0.963 & 386 & 0.00 & 0.10 & $-0.20,0.21$ & 0.974 \\
\hline \multicolumn{11}{|l|}{ Positive affect scores } \\
\hline \multicolumn{11}{|l|}{ Girls } \\
\hline Primary school & 221 & 0.11 & 0.10 & $-0.09,0.33$ & 0.289 & 212 & 0.12 & 0.10 & $-0.08,0.33$ & 0.253 \\
\hline Secondary school & 368 & 0.08 & 0.06 & $-0.03,0.21$ & 0.175 & 366 & 0.09 & 0.06 & $-0.03,0.21$ & 0.167 \\
\hline \multicolumn{11}{|l|}{ Boys } \\
\hline Primary school & 237 & 0.11 & 0.10 & $-0.09,0.31$ & 0.294 & 227 & 0.08 & 0.10 & $-0.12,0.29$ & 0.424 \\
\hline Secondary school & 388 & $0 \cdot 10$ & 0.06 & $-0.21,0.22$ & 0.105 & 388 & 0.09 & 0.06 & $-0.03,0.21$ & 0.143 \\
\hline \multicolumn{11}{|l|}{ Negative affect scores } \\
\hline \multicolumn{11}{|l|}{ Girls } \\
\hline Primary school & 223 & -0.16 & 0.10 & $-0.36,0.03$ & 0.109 & 215 & -0.14 & 0.10 & $-0.34,0.06$ & 0.174 \\
\hline Secondary school & 368 & -0.12 & 0.06 & $-0.21,-0.00$ & 0.068 & 366 & -0.11 & 0.06 & $-0.24,0.02$ & 0.097 \\
\hline \multicolumn{11}{|l|}{ Boys } \\
\hline Primary school & 237 & -0.09 & 0.09 & $-0.27,0.09$ & 0.346 & 228 & -0.12 & 0.09 & $-0.31,0.06$ & 0.208 \\
\hline Secondary school & 388 & -0.12 & 0.06 & $-0.25,0.01$ & 0.076 & 388 & -0.12 & 0.06 & $-0.25,0.01$ & 0.080 \\
\hline
\end{tabular}

$\beta$, Unstandardised coefficient.

* Model 1: adjusted for KIDSCREEN, positive affect or negative affect scores at baseline

† Model 2: adjusted for age, socio-economic status, prevalence of overweight and obesity (all collected at 2-year follow-up) and KIDSCREEN, positive affect or negative affect scores at baseline. 
study. The only improvement observed consisted of a decrease in negative affect in the primary school girls and boys. These results are difficult to interpret; however, they are partially consistent with the age and sex differences described in the literature examining well-being indicators and previously mentioned in this manuscript. Less favourable well-being has been shown with increasing age of the adolescents, particularly in female adolescent populations ${ }^{(31)}$. In particular, the tendency of a decreasing quality of life with age between childhood and adulthood has been reported previously ${ }^{(32)}$. Possible explanations could include hormonal changes, stressful life events and less efficacious coping strategies that emerge among girls in this age range ${ }^{(32)}$.

In this study, we found that MD adherence decreased over the 2-year follow-up for primary and secondary girls and boys. Relevant factors that could be associated with this change include an increase in screen time which has been associated with a poor diet ${ }^{(33)}$. Parental control is also known to change during this period, and parental control has been shown to influence dietary patterns ${ }^{(34)}$

\section{Potential reasons for the favourable effect of the Mediterranean diet adherence on well-being}

The mechanism explaining the favourable effect of MD adherence on well-being is still unknown. However, previous studies have found the MD to have beneficial effects on brain functioning $^{(8,35)}$, to act as a protective factor of neurodegenerative disease $^{(8)}$, and to reduce depressive symptoms in adolescent girls $^{(9,10)}$. Apart from the psychological mechanisms, biological mechanisms could also be involved. For example, there is evidence of an immune protective effect and anti-inflammatory properties associated with the $\mathrm{MD}^{(36)}$ as well as a protective effect on cardiovascular and metabolic diseases ${ }^{(11,12)}$

A current line of research that could help to elucidate the favourable effects of MD adherence on well-being in youth populations is one postulating diet as a modulator of gut microbiota. Previous studies have described the positive influence of the MD on gut microbiota ${ }^{(37,38)}$ as well as the beneficial link between the commensal microbiota on host physiology and brain development and function ${ }^{(39)}$. Alterations in gut microbiota have been shown to influence stressrelated behaviours and could be related to mood and anxiety disorders $^{(40)}$. It would seem reasonable to speculate that MD adherence may improve gut microbiota homoeostasis, which may in turn influence brain functioning and development, thus improving subjective well-being and HR-QoL of adolescent populations.

However, other factors might come in to play when considering the relationship between MD adherence and well-being, particularly contextual factors may determine child and adolescent's diet and well-being. Previous research has shown the influence of family meal frequency, home availability of foods and parental dietary habits to be related to dietary patterns and well-being of the adolescents. Greater frequency of family meals has been found to be positively associated with diet ${ }^{(41-43)}$ and well-being of adolescents ${ }^{(44)}$. Also, healthy food availability as well as maternal, paternal and peer support for healthy eating were positively associated with dietary patterns of the adolescents ${ }^{(41)}$. Parental reported intakes of fruits and vegetables were also associated with a greater quality of diet in children ${ }^{(45)}$.

In addition, family environment should be considered. The presence of family conflict may negatively influence dietary practices of adolescents ${ }^{(46)}$, and discrepancies among family members are related to well-being of adolescents ${ }^{(47)}$. Parental modeling, parental rules and parental encouragement seem to be related to eating behaviours of children and adolescents ${ }^{(48)}$. Along the same line, the family structure has been shown as a determinant of diet and well-being. Particularly, the cohabitation with both parents may positively influence diet ${ }^{(49)}$, while single-parent family background may relate to worse mental health in adolescents ${ }^{(50)}$. Finally, factors such as increased meal skipping and meal consumption away from home may also affect diet quality ${ }^{(51)}$.

There are limitations associated with this study which must be considered when interpreting the findings. With the exception of overweight/obese status, all variables were selfreported, which may influence the quality of data inherent to the use of questionnaires (although the questionnaires used are valid and reliable). It is possible that residual confounding remained as a result of unmeasured covariates or the presence of measurement error in those covariates included in the models. The cross-sectional nature of some of these data and lack of findings from the longitudinal analysis cannot infer causation, and we can only report associations between MD adherence and well-being. It is not known whether a higher MD adherence led to greater well-being or whether greater well-being led to a high MD adherence in this sample. Selfreported pubertal assessment is not a reliable measure of exact pubertal staging, although it has been frequently used in epidemiological studies due to its accuracy for a simple distinction between pre-puberty and puberty. The results of this study may not be generalisable to other populations beyond this sample of Spanish primary and secondary school girls and boys. Finally, future longitudinal studies with a longer followup should be carried out to extend the longitudinal perspective examined in this study. However, the present study has examined a wide sample of children and adolescents, considering several well-being indicators.

In conclusion, higher MD adherence was found to be associated with better well-being in a population of Spanish secondary school girls and boys. However, MD adherence at baseline did not predict well-being after 2-year follow-up. Further research including intervention studies are required to determine causality between MD adherence and well-being, which might help inform future public health nutrition programs for this target group.

\section{Acknowledgements}

The authors gratefully acknowledge the youth, families and teachers who participated in this study.

The UP\&DOWN study was supported by the DEP 201021662-C04-00 grant from the National Plan for Research, 
Development and Innovation $(\mathrm{R}+\mathrm{D}+\mathrm{i})$ MICINN. I. E.-C. is supported by a grant from the Alicia Koplowitz Foundation.

L. E.-G. was an investigator of the UP\&DOWN study, designed the study presented in the manuscript, carried out the statistical analyses and wrote the draft of the article. A. I. T. contributed to design the study presented in the manuscript, to carry out the statistical analysis, to the interpretation of the results obtained and she revised the article. S. J. T. contributed to design the study presented in the manuscript, to carry out the statistical analysis, to the interpretation of the results obtained and she revised the article. I. E.-C. was an investigator of the UP\&DOWN study and revised the article. She contributed in the data acquisition. J. C.-P. was a principal investigator of the UP\&DOWN study and revised the article. Á. D.-A. was an investigator of the UP\&DOWN study and revised the article. He contributed in the data acquisition. A. M. was a coordinator and main investigator of the UP\&DOWN study. She oriented and revised the article. S. G.-M. was an investigator of the UP\&DOWN study and revised the article. Ó. L. V. was a coordinator and main investigator of the UP\&DOWN study. He oriented and revised the article. All authors approved the final version of the manuscript.

The authors declare that there are no conflicts of interest.

\section{References}

1. Chida Y \& Steptoe A (2008) Positive psychological well-being and mortality: a quantitative review of prospective observational studies. Psychosom Med 70, 741-756.

2. Merikangas KR, Nakamura EF \& Kessler RC (2009) Epidemiology of mental disorders in children and adolescents. Dialogues Clin Neurosci 11, 7-20.

3. Diener E, Lucas RE \& Oishi S (2002) Subjective well-being. In Handbook of Positive Psychology, pp. 63-73 [CR Snyder and SJ López, editors]. Oxford/New York: Oxford University Press.

4. Watson D, Clark LA \& Tellegen A (1998) Development and validation of brief measures of positive and negative affect: the PANAS scales. J Pers Soc Psychol 54, 1063-1070.

5. The World Health Organization Quality of Life Assessment (WHOQOL) (1995) Position paper from the World Health Organization. Soc Sci Med 41, 1403-1409.

6. O'Neil A, Quirk SE, Housden S, et al. (2014) Relationship between diet and mental health in children and adolescents: a systematic review. Am J Public Health 104, e31-e42.

7. Bach-Faig A, Berry EM, Lairon D, et al. (2011) Mediterranean diet pyramid today. Science and cultural updates. Public Health Nutr 14, 2274-2284.

8. Anastasiou CA, Yannakoulia M, Kosmidis MH, et al. (2017) Mediterranean diet and cognitive health: initial results from the Hellenic Longitudinal Investigation of Ageing and Diet. PLOS ONE 12, e0182048.

9. Trichopoulou A, Bamia C \& Trichopoulos D (2009) Anatomy of health effects of Mediterranean diet: Greek EPIC prospective cohort study. BMJ 338, b2337.

10. Voltas N, Arija V, Aparicio E, et al. (2016) Longitudinal study of psychopathological, anthropometric and sociodemographic factors related to the level of Mediterranean diet adherence in a community sample of Spanish adolescents. Public Health Nutr 19, 1812-1822.

11. Babio N, Toledo E, Estruch R, et al. (2014) Mediterranean diets and metabolic syndrome status in the PREDIMED randomized trial. CMAJ 186, E649-E657.
12. Widmer RJ, Flammer AJ, Lerman LO, et al. (2015) The Mediterranean diet, its components, and cardiovascular disease. Am J Med 128, 229-238.

13. Costarelli V, Koretsi E \& Georgitsogianni E (2013) Healthrelated quality of life of Greek adolescents: the role of the Mediterranean diet. Qual Life Res 22, 951-956.

14. Muros JJ, Salvador Perez F, Zurita Ortega F, et al. (2017) The association between healthy lifestyle behaviors and health-related quality of life among adolescents. J Pediatr $\mathbf{9 3}$, 406-412.

15. Bolton KA, Jacka F, Allender S, et al. (2016) The association between self-reported diet quality and health-related quality of life in rural and urban Australian adolescents. Aust J Rural Health 24, 317-325.

16. Kulkarni AA, Swinburn BA \& Utter J (2015) Associations between diet quality and mental health in socially disadvantaged New Zealand adolescents. Eur J Clin Nutr 69, 79-83.

17. Grao-Cruces A, Nuviala A, Fernandez-Martinez A, et al. (2013) Adherence to the Mediterranean diet in rural and urban adolescents of southern Spain, life satisfaction, anthropometry, and physical and sedentary activities. Nutr Hosp 28, 1129-1135.

18. Castro-Piñero J, Carbonell-Baeza A, Martinez-Gomez D, et al. (2014) Follow-up in healthy schoolchildren and in adolescents with DOWN syndrome: psycho-environmental and genetic determinants of physical activity and its impact on fitness, cardiovascular diseases, inflammatory biomarkers and mental health; the UP\&DOWN Study. BMC Public Health 14, 400.

19. Serra-Majem L, Ribas L, Ngo J, et al. (2004) Food, youth and the Mediterranean diet in Spain. Development of KIDMED, Mediterranean Diet Quality Index in children and adolescents. Public Health Nutr 7, 931-935.

20. Muñoz CJ (2016) Adherencia a la dieta mediterránea en el alumnado de educación primaria en Málaga. (Adherence to the Mediterranean diet in primary school students of Malaga). Doctoral Dissertation, Málaga.

21. Sandín B (2003) PANAS scale of positive and negative affect for children and adolescents (PANASN). Rev Psicopatol Psicol Clin 8, 173-182.

22. Ravens-Sieberer U, Erhart M, Rajmil L, et al. (2010) Reliability, construct and criterion validity of the KIDSCREEN-10 score: a short measure for children and adolescents' well-being and health-related quality of life. Qual Life Res 19, 1487-1500.

23. KIDSCREEN Group (2006) Índice KIDSCREEN-10. https:// www.kidscreen.org/espa\%C3\%B1ol/cuestionario-kidscreen/ \%C3\%ADndice-kidscreen-10/ (accessed July 2018).

24. Currie C, Molcho M, Boyce W, et al. (2008) Researching health inequalities in adolescents: the development of the Health Behaviour in School-Aged Children (HBSC) family affluence scale. Soc Sci Med 66, 1429-1436.

25. Jimenez Pavon D, Ortega FP, Ruiz JR, et al. (2010) Socioeconomic status influences physical fitness in European adolescents independently of body fat and physical activity: the HELENA study. Nutr Hosp 25, 311-316.

26. Cole TJ, Bellizzi MC, Flegal KM, et al. (2000) Establishing a standard definition for child overweight and obesity worldwide: international survey. BMJ 320, 1240-1243.

27. Chavarro JE, Watkins DJ, Afeiche MC, et al. (2017) Validity of self-assessed sexual maturation against physician assessments and hormone levels. J Pediatr 186, 172-178.

28. Henriquez Sanchez P, Ruano C, de Irala J, et al. (2012) Adherence to the Mediterranean diet and quality of life in the SUN Project. Eur J Clin Nutr 66, 360-368.

29. Milte CM \& McNaughton SA (2016) Dietary patterns and successful ageing: a systematic review. Eur J Nutr $\mathbf{5 5}$, 423-450. 
30. Milte CM, Thorpe MG, Crawford D, et al. (2015) Associations of diet quality with health-related quality of life in older Australian men and women. Exp Gerontol 64, 8-16.

31. Cavallo F, Dalmasso P, Ottova-Jordan V, et al. (2015) Trends in life satisfaction in European and North-American adolescents from 2002 to 2010 in over 30 countries. Eur J Public Health 25, Suppl. 2, 80-82.

32. Michel G, Bisegger C, Fuhr DC, et al. (2009) Age and gender differences in health-related quality of life of children and adolescents in Europe: a multilevel analysis. Qual Life Res 18, $1147-1157$.

33. Falbe J, Willett WC, Rosner B, et al. (2014) Longitudinal relations of television, electronic games, and digital versatile discs with changes in diet in adolescents. Am J Clin Nutr $\mathbf{1 0 0}$, 1173-1181.

34. Dickens E \& Ogden J (2014) The role of parental control and modelling in predicting a child's diet and relationship with food after they leave home. A prospective study. Appetite 76, 23-29.

35. Feart C, Samieri C \& Barberger-Gateau P (2015) Mediterranean diet and cognitive health: an update of available knowledge. Curr Opin Clin Nutr Metab Care 18, 51-62.

36. Casas R, Sacanella E \& Estruch R (2014) The immune protective effect of the Mediterranean diet against chronic lowgrade inflammatory diseases. Endocr Metab Immune Disord Drug Targets 14, 245-254.

37. Del Chierico F, Vernocchi P, Dallapiccola B, et al. (2014) Mediterranean diet and health: food effects on gut microbiota and disease control. Int J Mol Sci 15, 11678-11699.

38. Lopez-Legarrea P, Fuller NR, Zulet MA, et al. (2014) The influence of Mediterranean, carbohydrate and high protein diets on gut microbiota composition in the treatment of obesity and associated inflammatory state. Asia Pac J Clin Nutr 23, 360-368.

39. Zhou L \& Foster JA (2015) Psychobiotics and the gut-brain axis: in the pursuit of happiness. Neuropsychiatr Dis Treat $\mathbf{1 1}$ $715-723$.

40. Foster JA \& McVey Neufeld KA (2013) Gut-brain axis: how the microbiome influences anxiety and depression. Trends Neurosci 36, 305-312.
41. Cutler GJ, Flood A, Hannan P, et al. (2011) Multiple sociodemographic and socioenvironmental characteristics are correlated with major patterns of dietary intake in adolescents. J Am Diet Assoc 111, 230-240.

42. Fink SK, Racine EF, Mueffelmann RE, et al. (2014) Family meals and diet quality among children and adolescents in North Carolina. J Nutr Educ Behav 46, 418-422.

43. Befort C, Kaur H, Nollen N, et al. (2006) Fruit, vegetable, and fat intake among non-Hispanic black and non-Hispanic white adolescents: associations with home availability and food consumption settings. J Am Diet Assoc 106, $367-373$.

44. Utter J, Denny S, Robinson E, et al. (2013) Family meals and the well-being of adolescents. I Paediatr Child Health 49. 906-911.

45. Santiago-Torres M, Adams AK, Carrel AL, et al. (2014) Home food availability, parental dietary intake, and familial eating habits influence the diet quality of urban Hispanic children. Child Obes 10, 408-415.

46. Hinchliff GLM, Kelly AB, Chan GCK, et al. (2016) Risky dieting amongst adolescent girls: associations with family relationship problems and depressed mood. Eat Behav 22, 222-224.

47. Stuart J \& Jose PE (2012) The influence of discrepancies between adolescent and parent ratings of family dynamics on the well-being of adolescents. I Fam Psychol 26, $858-868$

48. Pearson N, Biddle SJ \& Gorely T (2009) Family correlates of fruit and vegetable consumption in children and adolescents: a systematic review. Public Health Nutr 12, 267-283.

49. Papadaki S \& Mavrikaki E (2015) Greek adolescents and the Mediterranean diet: factors affecting quality and adherence. Nutrition 31, 345-349.

50. Laukkanen M, Hakko H, Riipinen P, et al. (2016) Does family structure play a role in depression in adolescents admitted to psychiatric inpatient Care? . Child Psychiatry Hum Dev 47, 918-924.

51. Storey KE, Hanning RM, Lambraki IA, et al. (2009) Determinants of diet quality among Canadian adolescents. Can J Diet Pract Res 70, 58-65. 